\title{
Fluid-Structure Simulation of a General Non-Contact Tonometry. A Required Complexity?
}

\author{
Miguel Ángel Ariza-Gracia ${ }^{\mathrm{a}, \mathrm{e}}$, W. Wu $\mathrm{Wu}^{\mathrm{b}}$, B. Calvo ${ }^{\mathrm{a}, \mathrm{c}}$, M. Malvèd, P. Büchler ${ }^{\mathrm{e}}$, José Felix Rodriguez Matas ${ }^{\mathrm{f}}$ \\ ${ }^{a}$ Aragón Institute of Engineering Research (i3A). Univesrity of Zaragoza (Zaragoza, Spain) \\ ${ }^{b}$ Department of Mechanical Engineering University of Texas at San Antonio (San Antonio, Texas, USA) \\ ${ }^{c}$ CIBER de Bioingeniería, Biomateriales y Nanomedicina (CIBER-BBN), Aragon Health Sciences Institute, Spain \\ ${ }^{d}$ Department of Mechanical Engineering, Public University of Navarra (Pamplona, Navarra, Spain) \\ ${ }^{e}$ Institute for Surgical Technology and Biomechanics (ISTB). Universität Bern (Bern, Switzerland) \\ ${ }^{f}$ LaBS, Department of Chemistry, Materials and Chemical Engineering "Giulio Natta”, Politecnico di Milano, Milano, Italy
}

\begin{abstract}
Understanding corneal biomechanics is important for applications regarding refractive surgery prediction outcomes and the study of pathologies affecting the cornea itself. In this regard, non-contact tonometry (NCT) is gaining interest as a non-invasive diagnostic tool in ophthalmology, and is becoming an alternative method to characterize corneal biomechanics in vivo. In general, identification of material parameters of the cornea from a NCT test relies on the inverse finite element method, for which an accurate and reliable modelization of the test is required. This study explores four different modelling strategies ranging from pure structural analysis up to a fluid-structure interaction model considering the air-cornea and humour-cornea interactions. The four approaches have been compared using clinical biomarkers commonly used in ophthalmology. Results from the simulations indicate the importance of considering the humors as fluids and the deformation of the cornea when determining the pressure applied by the air-jet during the test. Ignoring this two elements in the modeling lead to an overestimation of corneal displacement and therefore an overestimation of corneal stiffness when using the inverse finite element method.
\end{abstract}

Keywords: Corneal Biomechanics, Dynamic simulations, Fluid-structure interaction simulations, Non-contact tonometry 


\section{Introduction}

Elevated intraocular pressure (IOP) is responsible for the damage of the optical nerve. Prolonged exposition to this mechanical load leads to glaucoma and, eventually, to blindness. Since this degenerative process is generally asymptomatic, a number of devices have been developed to monitor the ocular pressure [1, 2, 3]. The first developed devices measured the IOP by direct contact with the cornea e.g. the Goldman tonometer, whereas the new generation of non-contact tonometers (NTC), are able to measure the IOP without direct contact with the cornea. Non-contact tonometers rely on a short air pulse to induce a deformation of the cornea. During corneal deformation, a twodimensional transversal slice is recorded using a Scheimpflug camera (see Fig. 1.a) from which the IOP pressure is calculated. In addition to the estimation of the patient's IOP, these devices provide with additional mechanical markers derived from the evolution of the corneal curvature over the time of the test $[4,5,6]$. Some of these markers are: i) the deformation amplitude (see in Fig. 1.a1), ii) the first and second applanation times and lengths (see in Fig. 1.a2), iii) the corneal speed (see in Fig. 1.a3), and iv) the corneal hysteresis (see in Fig. 1.b). These mechanical markers aim at being used to assess the result of a refractive surgery [7] or to evaluate the evolution of certain pathologies such as keratoconus [8].

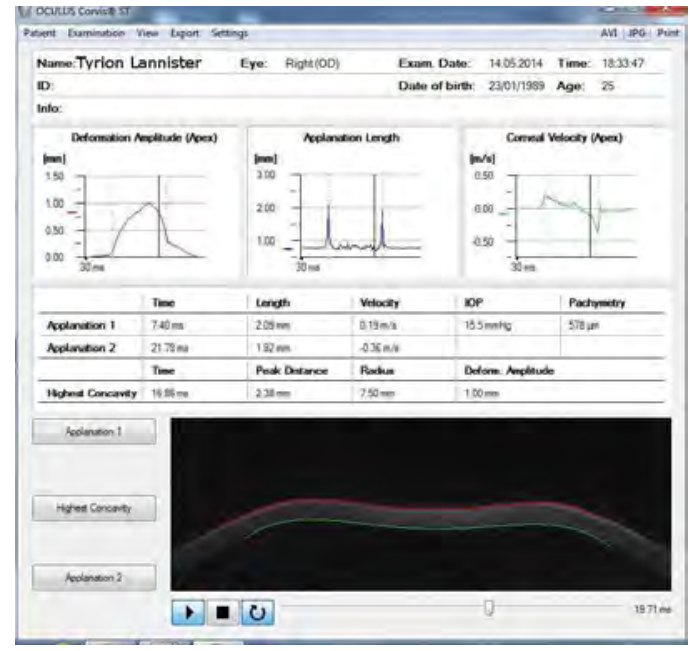

(a)

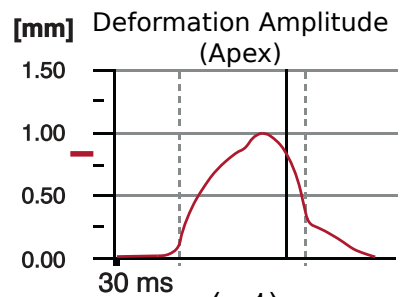

(a.1)

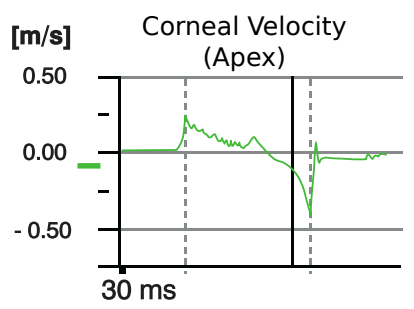

(a.3)

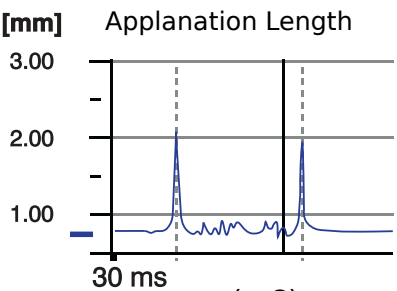

(a.2)

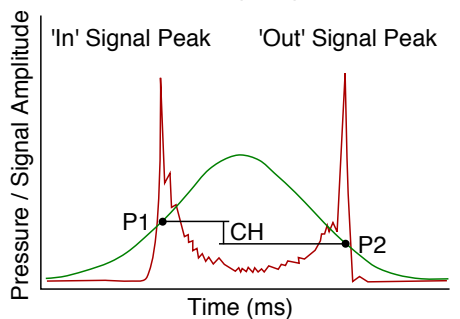

(b)

Figure 1: Mechanical markers measured with non-contact tonometers: CorVis and Ocular Response Analyser (ORA): (a) Capture of CorVis's Graphic User Interface. Based on medical imaging (corneal section in the bottom), different biomarkers are measured: (a.1) deformation amplitude, (a.2) applanation length and time, (a.3) corneal velocity. The deformation amplitude measures the displacement of the apex over time, the applanation length gives the greatest flattened area of the cornea when going inwards/outwards and the time at which they happened, and the corneal velocity gives the velocity of the apex over time; (b) Corneal Hysteresis (Orbscan) is the difference between the pressure measured at the first applanation (going inwards) and the second applanation (going outwards) $\left(\mathrm{CH}=\mathrm{P}_{1}-\mathrm{P}_{2}\right)$.

The mechanical response of the ocular system during a NCT measurement is complex. The process relies on an air pulse of amplitude 5 to 8 times the physiological IOP, of the order of $15 \mathrm{mmHg}$, and duration of approximately $30 \mathrm{~ms}[4,5,6,9]$. The mechanisms responsible for the load transfer from the air jet to the cornea are complicated as they depend on the patient's anatomy and position, the intraocular pressure, and the mechanical properties of the cornea among others. In addition, the fast corneal loading due to the air-puff induces a complex dynamic response of the eyeball. For these reasons, the measurement of the IOP for clinical use is not directly provided by a mechanistic analysis of the NCT test, but is determined indirectly through empirical correlations between the measured deformation profile of the cornea and known intraocular pressure measurements [10]. In addition, the mechanical markers currently obtained with this method lack of a direct physical interpretation and their use for the treatment of patients should be handled with care.

Despite of the limitations, non-contact tonometry is appealing to obtain a patient-specific characterization of the mechanical condition of the eye in vivo. However, a deeper understanding of the mechanisms, and their interactions, 
involved in the test is required in order to correctly translate these results to the clinic, or to instruct computational models used for planning refractive interventions.

Few studies have been performed to simulate a non contact tonometry test. To date, most studies have been limited to structural analysis considering the mechanical response of the cornea only, neglecting the internal structures in most cases, and ignoring the fluid-structure interaction of the air with the cornea during the test. Kling et al. [11] used twodimensional CFD simulations with different corneal deformations to determine the pressure applied to the cornea due to the air-puff at different stages of the deformation. The resulting pressure profile was subsequently used to perform a dynamic simulation of the cornea considering a constant IOP and ignoring the effect of the humors and internal structures. Similarly, Roy et al. [12] also used two-dimensional CFD simulations on underformed cornea to determine the time-varying pressure profile acting on the cornea. They suggest that FSI simulations were not needed due to the prevalence of normal forces with respect to shear forces. Ariza-Gracia et al. [9, 13, 14] have considered a three dimensional model of the eyeball in simulating the NCT test. Even though the anisotropy of the cornea is accounted for, internal structures and humors are ignored and substituted by a constant IOP. The pressure profile due to the air-puff is also found by means of CFD simulations as in [12]. Their results highlight that NCT's measurements are an interplay of different effects (geometry, stiffness and pressure). Simonini et al. $[15,16]$ applied an analytic bellshaped pressure distribution over the cornea, and suggested that the viscoelastic properties of the cornea were prone to play a minor role in comparison to the elastic properties of the cornea. Recently, Montanino et al. [6] carried out the first simulation of a NCT test considering the presence of the aqueous humor. They demonstrated the importance of considering the aqueous humor as a fluid showing that, IOP does not remain constant during a NCT test. However, they do not account for the air-cornea fluid structure interaction.

To date, and to the best of our knowledge, the few FSI simulations regarding the eyeball are related to the analysis of eyeball injuries [17, 18], or to the measure of IOP [19]. However, there are not FSI simulations of the NCT test. Despite the interest of all these studies, the proper interaction between the air and the cornea still remains unknown. This study aims at exploring different approaches to simulate a NCT test. Four modelling strategies ranging from a pure structural analysis (including some internal features of the eye) up to a fully coupled fluid-structure interaction model (air-cornea and humour-cornea) are considered. The different approaches have been systematically compared using clinical biomarkers in order to test the performance of each one.

\section{Material and Methods}

Four models have been developed to evaluate different simulation strategies of a non-contact tonometry test (Fig. 2). In increasing order of complexity, the developed models are:

- Case 1 structural model without internal structures and humors modeled as uniform pressure;

- Case 2 structural model without internal structures and humors modeled as fluid without mass (fluid cavity);

- Case 3 structural model with internal structures (lens and ciliary muscles) and humors modeled as fluid without mass;

- Case 4 fluid-structure interaction model with internal structures and humors modeled as fluid with mass (lagrangian element). Air in contact with the cornea is considered as an incompressible fluid for the FSI analysis.

For the sake of comparison, Case 3 in Fig. 2 has been considered as a reference model. In this regard, material parameters of the cornea were calibrated to match typical NCT experimental results using this model. The models were evaluated based on the deformation of the cornea from which a number of clinical biomarkers were calculated, namely: first and second applanation time and length, corneal velocity at first and second applanation time, and corneal hysteresis. To establish a non-biased comparison, the same geometry, mesh, and material behavior were used for the eyeball in all models.

\subsection{Geometrical and mechanical description}

A 2D-axisymmetric model of an average porcine eye was built. The main ocular structures were included in the model (Fig. 3), namely: the cornea with a constant thickness of 850 microns, the sclera with an average thickness of $1 \mathrm{~mm}$, the crystalline lens, and the ciliary muscles. 


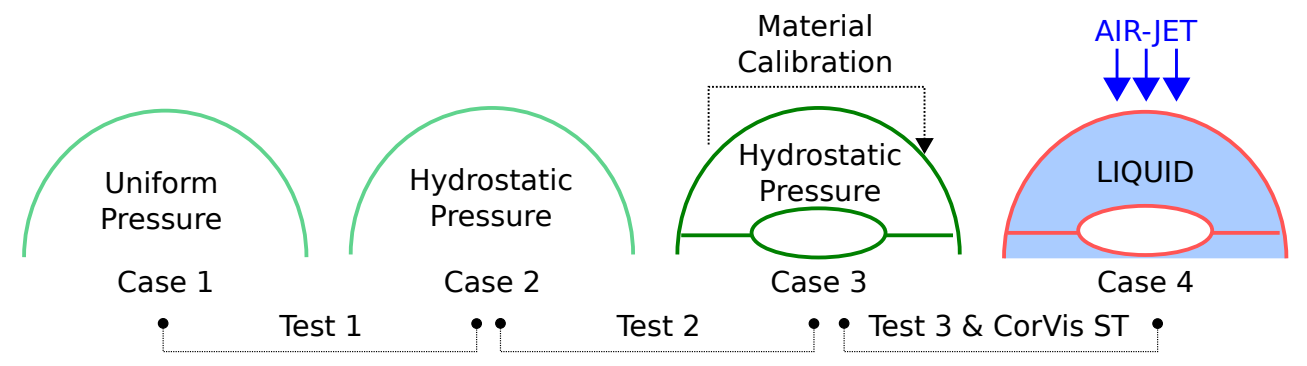

Figure 2: Benchmark models. Four models were used to evaluate various simulation strategies. Three models were focused on dynamic structural simulations (Green) and one on FSI simulations (Red). These models included different approaches to simulate the intra-ocular pressure and the presence (or not) of internal structures. Case 3 was used to calibrate the material parameters to match experimental measurement of NCT tests. Three tests were used to compare the models: i) Test 1 to compare a uniform internal pressure to simulate the effect of the humors against modeling the humors as a fluid cavity; ii) Test 2 to compare the effect of the internal structures on the corneal deformation; iii) Test 3 to assess the importance of conducting a fully coupled FSI simulation to model a NCT test.

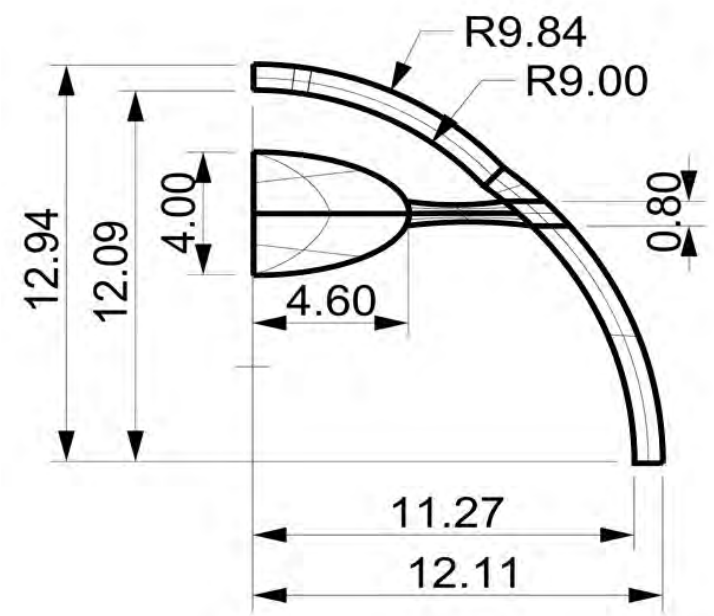

(a)

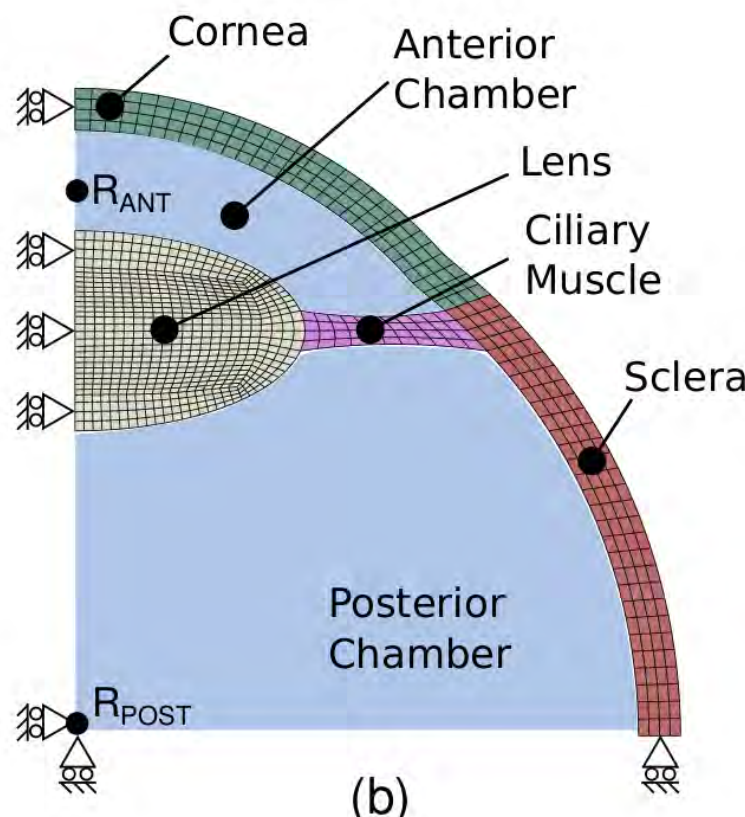

(b)

Figure 3: Geometry and structures of the numerical model. (a) Dimensions of the average porcine model in millimeters; (b) Anatomy of the ocular structures, mesh, and boundary conditions of the model. BCs are restrained along vertical and horizontal axes. $R_{A N T}$ and $R_{P O S T}$ are the reference points used to pressurize the cavities. 


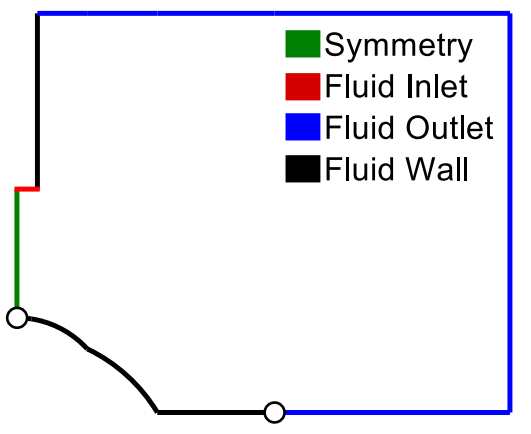

(a)

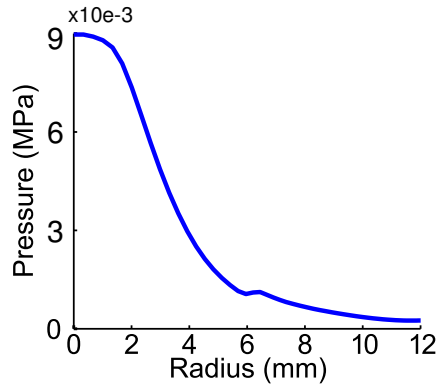

(b)

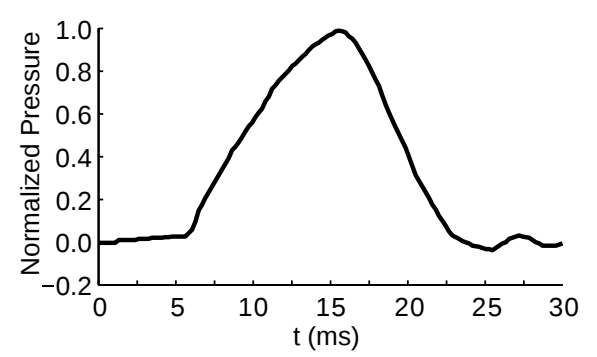

(c)

Figure 4: Definition of the fluid boundary conditions. (a) Boundary conditions of the fluid mesh (LS-DYNA). The fluid wall condition for the eyeball was slightly extended in the horizontal plane to include the effect of the cheek on the flow pattern; (b) Spatial profile of pressure over the cornea determined with LS-DYNA. Cornea was assumed as a rigid wall. The total force applied by the distributed pressure is $8.5 \mathrm{~N}$; (c) Temporal evolution of the air pressure in Pentacam (provided by Oculus).

The mechanical behavior of the sclera was model using a Yeoh-type strain energy function as proposed in literature $[9,14,13,20]$

$$
\psi_{Y}=\sum_{i=1}^{3} Y_{i 0}\left(\tilde{I}_{1}-3\right)^{i}+\sum_{i=1}^{3} \frac{1}{D_{k_{i}}}(J-1)^{2 i}
$$

where $Y_{i 0}(\mathrm{MPa})$ are the material constants associated with the sclera behavior and $D_{k_{i}}\left(\mathrm{MPa}^{-1}\right)$ determines the compressibility of the material. $J=\operatorname{det} \mathbf{F}$ characterises the volume-changing part of the deformation and $\tilde{I}_{1}$ is the first invariant of the modified right Cauchy-Green tensor $\left(\tilde{\mathbf{C}}=J^{-2 / 3} \mathbf{F}^{T} \mathbf{F}\right)$. The crystalline lens and the muscle were assumed to be linear elastic [19]. The fluid properties of the humors correspond to water in normal conditions; incompressible without thermal expansion, and having a density of $1000 \mathrm{~kg} / \mathrm{m}^{3}$. The mechanical parameters of the model are reported in table 1.

As previously reported $[13,21]$, the material behavior of the cornea was defined by a Demiray strain energy function [22] to account for its nonlinear stress/strain relationship;

$$
\psi_{D}=D_{1}\left(e^{D_{2}\left(\tilde{I}_{1}-3\right)}-1\right)+\frac{1}{D_{k}}(J-1)
$$

where $D_{1}(\mathrm{MPa})$ and $D_{2}(-)$ are material parameters, and $D_{k}\left(\mathrm{MPa}^{-1}\right)$ determines the compressibility of the material. The material parameters of the cornea $\left(D_{1}, D_{2}\right)$ were identified using a grid-search optimization based on the most complex structural model (case 3 in Fig.2). The sequential grid-search seeks to minimize the average response of ex-vivo inflation experiments [23] and NCT tests [24]. The optimization was performed in two steps, first a coarse optimization was performed based on initial conditions taken from literature. In a second step, a finer search is perform in the neighborhood of the first minimum [25]. The optimization procedure was setup in Python 3.6 [26]. The optimal material parameters are given in table 1 with Fig. 5 showing the contour plot of the objective function around the optimum indicating the significant sensitivity to changes in $D_{2}$.

The built-in material models in Abaqus and LS-DYNA were used to model the sclera, the ciliary muscles and the cristaline lens. However, strain energy function in Eq. 2 was implemented in a user material subroutine in both softwares.

\subsection{Structural simulations}

For the structural models Case 1, Case 2 and Case 3, the simulation of the NCT was performed into two steps using Abaqus/Explicit (Dassault Systèms). First, the eyeball was inflated with a physiological intraocular pressure of $15 \mathrm{mmHg}$. The duration of the step was $100 \mathrm{~ms}$ to avoid any dynamic effects. The second step corresponded to the application of an air pulse on the anterior surface of the cornea. The spatial-temporal pressure distribution due 


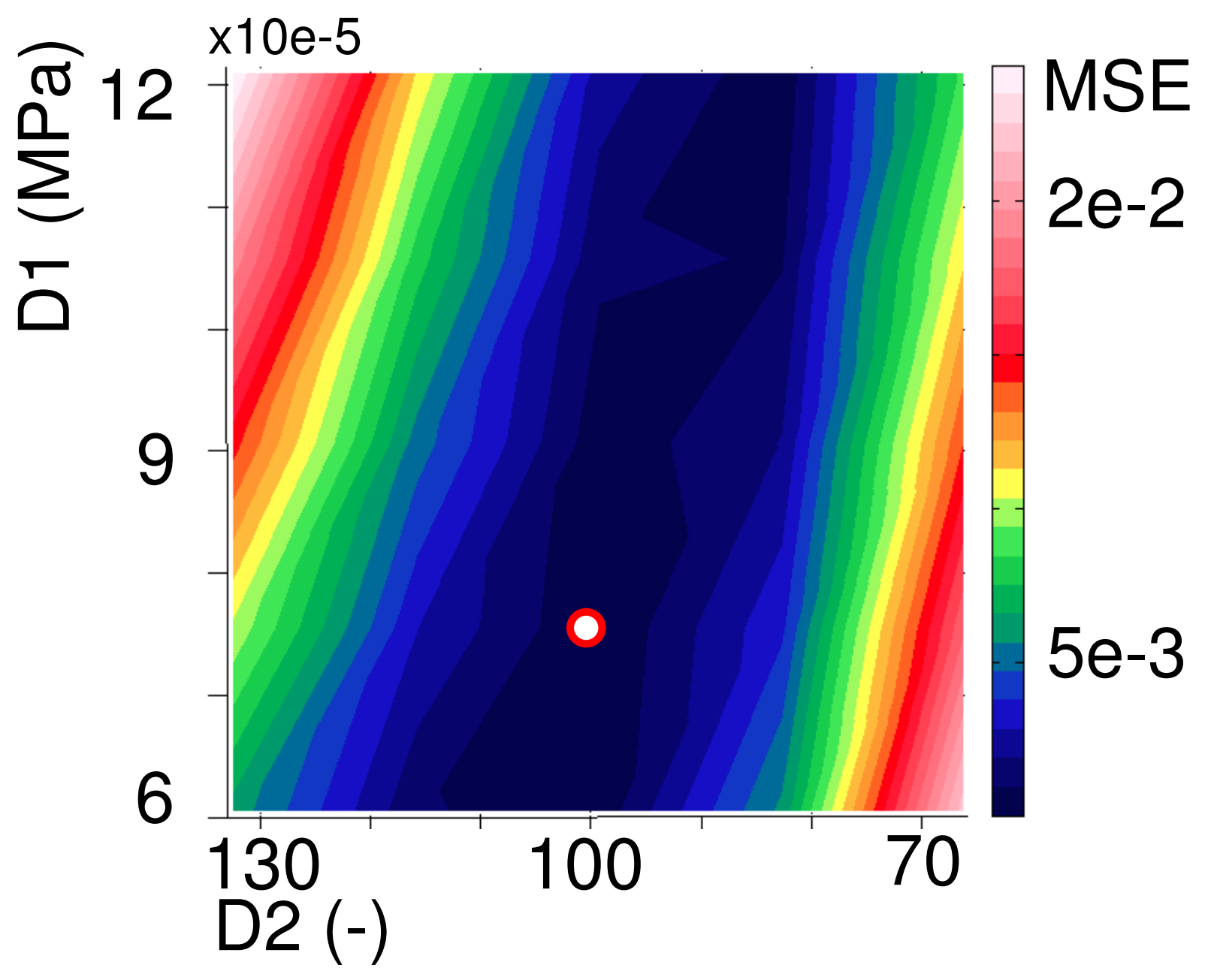

Figure 5: Material grid calibration. Mean squared error between real $(U=1.2 \mathrm{~mm})$ and numerical corneal displacement (MSE) with respect to the material parameters $\left(D_{1}, D_{2}\right)$ obtained with the second fine grid-search optimization and the material calibration model (see case 3 in Figure 2$)$. The minimum is marked as a red and white dot.

\begin{tabular}{c|cccccc} 
& \multicolumn{7}{c}{ Table 1: Material Parameters of the Ocular Tissues } \\
Cornea & $D_{1}(\mathrm{MPa})$ & $D_{2}(-)$ & $1 / D_{k}(\mathrm{MPa})$ & $\mu(\mathrm{kg} /(\mathrm{m} \cdot \mathrm{s}))$ & $\rho\left(\mathrm{kg} / \mathrm{m}^{3}\right)$ & \\
& $7.6 \cdot 10^{-5}$ & 99.2 & $10^{7}$ & $1.81 \times 10^{-5}$ & 1,225 & \\
\hline & $Y_{10}(\mathrm{MPa})$ & $Y_{20}(\mathrm{MPa})$ & $Y_{30}(\mathrm{MPa})$ & $1 / D_{k_{i}}(\mathrm{MPa})$ & $\mu(\mathrm{kg} /(\mathrm{m} \cdot \mathrm{s}))$ & $\rho\left(\mathrm{kg} / \mathrm{m}^{3}\right)$ \\
Sclera [14] & 0.8 & 56.1 & 2332.3 & $10^{7}$ & $1.81 \times 10^{-5}$ & 1,225 \\
\hline & $E(\mathrm{MPa})$ & $v(-)$ & $\mu(\mathrm{kg} /(\mathrm{m} \cdot \mathrm{s}))$ & $\rho\left(\mathrm{kg} / \mathrm{m}^{3}\right)$ & & \\
Lens [19] & 1.45 & 0.47 & $1.81 \times 10^{-5}$ & 1,225 & & \\
\hline & $E(\mathrm{MPa})$ & $v(-)$ & $\mu(\mathrm{kg} /(\mathrm{m} \cdot \mathrm{s}))$ & $\rho\left(\mathrm{kg} / \mathrm{m}^{3}\right)$ & & \\
Muscle [19] & 0.35 & 0.47 & $1.81 \times 10^{-5}$ & 1,225 &
\end{tabular}


to the air-puff was determined from a transient CFD simulation. The CFD model assumed a rigid corneal surface (i.e. modeled as a wall) as shown in Fig. 4.a, and a velocity profile, as shown in Fig. 4.b, with a peak velocity of $120 \mathrm{~m} / \mathrm{s}$ was defined at the fluid inlet (see Fig. 4.a). Parameters of the CFD simulation were identical to those used for the FSI simulation detailed in the following section. The spatial distribution of the fluid pressure on the corneal wall was recorded at each time step (Fig. 4.b shows the spatial profile of the pressure at the moment of maximum apical pressure) and used as a pressure load applied on the anterior corneal surface in the structural simulations. A damping coefficient, $\alpha$, of 0.1 was used to avoid unrealistic dynamic oscillations.

Symmetry boundary conditions were set along the vertical axis (corresponding to the axi-symmetric axis) and on the horizontal plane where only radial expansion of the eyeball is permitted (Fig.3.b). Two different strategies were considered to model the aqueous and the vitreous humors. First, a distributed surface load was applied on the cavities of the eyeball (Case 1, Fig.2). A second strategy consisted on defining a hydrostatic pressure condition in both cavities of the eyeball to constrain the volume of the cavity to remain constant (Case 2 and Case 3, Fig.2). This constrain was ensured by modeling the cavities of the eyeball as fluid cavities in Abaqus. In both cases, the initial intraocular pressure was set to a physiological value of $15 \mathrm{mmHg}$.

The solid mesh corresponding to the eyeball structures was composed of 957 4-nodes axisymmetric elements with reduced integration (CAX4R) and hourglass control. A sensitivity analysis was conducted to determine the appropriate mesh size.

\subsection{Fluid-structure simulations}

Fluid structure simulations of the NCT test were performed using the commercial finite element solver LS-DYNA Release 9.0. The structural mesh used for Cases 1 through 3 was directly imported to LS-DYNA. The characteristic dimension of the fluid domain was set 10 times larger than the characteristic dimension of the eye model (i.e. the diameter of the eyeball) so as to minimise boundary effects on the flow over the cornea. Air has been treated as an incompressible newtonian fluid $\left(\rho=1.177 \mathrm{Kg} / \mathrm{m}^{3}, \mu=0.018 \mathrm{cP}\right)$. The incompressible fluid solver ICFD from LSDYNA was used to solve for the air domain with a variational multiscale turbulent model. In addition, due to the large differences in mass density between the air and the eye (three orders of magnitude), a weak coupling for the FSI was considered. The fluid mesh corresponding to air was meshed with triangular elements using an adaptive meshing algorithm implemented within ICFD that, automatically refine the domain in order to maintain the accuracy of the solution. Average mesh sizes in the fluid domain were of the order of 23000 triangular elements

The aqueous and vitreous humors present in the anterior and posterior chambers were modelled using Lagrangian elements exhibiting non deviatoric stress-strain response i.e., $\sigma=P \mathbf{1}$, with the hydrostatic pressure described by a Mie-Grüneisen equation of state of the form,

$$
P=\frac{\rho_{0} C^{2} \mu\left[1+\left(1-\gamma_{0} / 2\right) \mu\right]}{\left[1+\left(S_{1}-1\right) \mu-S_{2} \frac{\mu^{2}}{\mu+1}-S_{3} \frac{\mu^{3}}{(\mu+1)^{2}}\right]^{2}}+\gamma_{0} E,
$$

where $P$ is the hydrostatic pressure in $P a, \rho_{0}$ is the initial density of water, $\rho=1000 \mathrm{~kg} / \mathrm{m}^{3}, C$ is the speed of sound in water, $C=1520 \mathrm{~m} / \mathrm{s}, \gamma_{0}=0.35$ is the Grüneisen gamma, $S_{1}=1.92, S_{2}=-0.096, S_{3}=0.0$ are dimensionless coefficients, and $E$ is the internal energy [27]. The anterior and posterior chambers were meshed with 370 and 1,044 quadrilateral elements respectively. Similarly to the structural part, a damping coefficient $(\alpha=0.1)$ was used.

Regarding the boundary conditions for the fluid, a fully developed condition was assumed at the air inlet coincident with the nozzle outlet of the tonometer located at $11 \mathrm{~mm}$ from the corneal apex, non-slip boundary conditions were set at eyeball surface in contact with air, and zero pressure outflow conditions for the remaining boundaries of the fluid domain (red, black, and blue lines, respectively, in Figure 4.a). A velocity profile as shown in Fig. 4.c with a peak velocity of $120 \mathrm{~m} / \mathrm{s}$ was defined at the fluid inlet.

\subsection{Simulation workflow and data analysis}

Three different tests were conducted to compare the different modeling strategies (see Fig. 2): i) Test 1 compares models without inner structures (lens and ciliary muscle) imposing a constant IOP against accounting for the presence of the humors (modeled as fluid cavity); ii) Test 2 to compare the effect of the internal structures on the corneal deformation with humors treated as fluid cavity, and iii) Test 3 was used to assess the importance of conducting 
fully coupled FSI simulations to model a NCT test. Both structural and FSI models used in Test 3 account for inner structures and model the humors as a fluid.

During a NCT's air pulse, cornea goes through 5 main stages: $i$ ) the initial steady position from where the cornea goes inwards, ii) the first applanation where a 3-mm central area of the cornea is analyzed to determine how much is flattened, iii) the maximum concavity where the cornea reaches its highest deformation amplitude, iv) the second applanation, and, $i v$ ) back to the initial position. Based on this corneal deformation, the following corneal biomarkers were analyzed: i) the maximum deformation amplitude (DA) of the corneal apex; ii) the evolution of the IOP during the test; iii) the corneal speed at first and second applanation times; iv) the applanation times and lengths; and v) the corneal hysteresis $(\mathrm{CH})$ defined as the difference in air pressures between first applanation (P1) and the second applanation (P2), or (P1 - P2), as shown in Fig. 1.b. Corneal hysteresis is considered in order to demonstrate the impact of considering the humors in the model.

\section{Results}

Case 3 in Fig. 2 was used to calibrate the material parameters of the cornea, and was taken as the golden standard to compare the performance of the other models. The maximum deformation amplitude obtained with this model was comparable to typical values of NCT (see blue row in table 2). As expected, the maximal displacement included in the optimization cost function was accurately reproduced by the numerical model (error of less than $1 \%$ ). While for Case 4, the remaining biomarkers were also in the experimental range observed on patients, in Case 3 were close but not in the experimental range. For example, the 1st App. time and length are in the upper bound, the 2nd. App. V, the 2nd App. time and amplitude were slightly below the lower bound of the experimental range (see in table 2). Only the corneal hysteresis greatly differed from the experimental data, resulting in an hysteresis about two times higher than the experimental range.

\begin{tabular}{|c|c|c|c|c|c|}
\hline Parameter & Case 1 & Case 2 & Case 3 & Case 4 & Exp. Ranges \\
\hline 1st App. Time (ms) & 5.25 & 7.25 & 10.4 & 9.3 & $7.40-8.27$ \\
\hline 1st App. Length (mm) & 1.7 & 1.3 & 2.2 & 1.1 & $1.47-2.18$ \\
\hline 1st App. V (mm/ms) & -0.37 & -0.17 & -0.14 & -0.20 & -0.15 to -0.09 \\
\hline 1st App. Pressure $(\mathrm{kPa})$ & 0.3 & 2.3 & 5.6 & 4.5 & - \\
\hline Delay (ms) & 0.25 & 0.25 & 0.30 & 0.73 & - \\
\hline Max. DA (mm) & 2.85 & 1.38 & 1.19 & 0.41 & $0.92-1.36$ \\
\hline Max. DA V (mm/ms) & 0.015 & 0.02 & 0.01 & 0.05 & - \\
\hline Max. DA Press. (kPa) & 8.98 & 8.98 & 8.97 & 8.91 & - \\
\hline 2nd App. Time (ms) & 25.50 & 23.25 & 20.8 & 20.0 & $22.05-23.13$ \\
\hline 2nd App. Length (mm) & 1.46 & 1.70 & 1.13 & 0.79 & $1.17-2.63$ \\
\hline 2nd App. V (mm/ms) & 0.38 & 0.16 & 0.21 & 0.27 & $0.25-0.44$ \\
\hline 2nd App. Press. (kPa) & -0.2 & 0.2 & 2.6 & 3.7 & - \\
\hline $\mathrm{CH}(\mathrm{mmHg})$ & 3.5 & 15.9 & 22.5 & 6.2 & $7.9-10.7$ \\
\hline Max. IOP ant $(\mathrm{kPa})$ & $-^{a}$ & 4.9 & 5.2 & 4.6 & - \\
\hline Max. IOP post $(\mathrm{kPa})$ & $-^{a}$ & $--^{b}$ & 4.7 & 3.3 & - \\
\hline Vol. ant. $\left(\mathrm{mm}^{3}\right)$ & $-^{a}$ & 3158.3 & 319.1 & $-^{c}$ & $-^{d}$ \\
\hline Vol. post. $\left(\mathrm{mm}^{3}\right)$ & $-^{a}$ & $-^{b}$ & 2576.1 & $-^{c}$ & $-^{d}$ \\
\hline
\end{tabular}

Notes: ${ }^{a}$ Case 1 did not provide with these information; ${ }^{b}$ Case 2 only has one chamber encompassing anterior and posterior chamber; ${ }^{c}$ Same volume as Case 3; ${ }^{d}$ The average volume of anterior chamber is $300 \mathrm{~mm}^{3}$ [30], and the average total volume of the eye is $6500 \mathrm{~mm}^{3}$ [31]. Due to the scleral symmetry, approximately the half of the volume is achieved.

Regarding the structural models, Table 2 shows that Case 3 results stiffer in terms of the apical displacement than the structural models Case 1 and Case 2. The increase in stiffness is in part due to the presence of internal structures (lens and ciliary muscles) as evidenced from the results from Case 2 and Case 3 in Table 2 (a 16\% difference in the apical displacement). However, modeling the humors as a fluid cavity instead of a constant IOP has a much more 
significant effect on the maximal apical displacement. Assuming a constant IOP (Case 1) resulted in a maximal apical displacement twice as large as for Case 2 where the posterior chamber has been modeled as a fluid cavity. This difference in the apical displacement increases to 2.5 times when compared with the golden standard Case 3, and up to 7 times for the FSI model Case 4. Regarding corneal hysteresis $(\mathrm{CH})$, considering a constant IOP in the chambers resulted in a $\mathrm{CH}$ about 6 times lower with respect to the reference model. Note that, structural models with the fluid cavity and internal structures overestimate $\mathrm{CH}$, whereas the model with a constant IOP underestimates it. In this regard, the FSI model yield a $\mathrm{CH}$ closer to the experimental range. On the contary, Cases 1 and 2 showed better accuracy in reproducing secondary dynamic variables such as applanation times and lengths, but not in reproducing corneal velocity.

The FSI model presented a maximum deformation amplitude about 3 times smaller than the golden standard (table 2). The intraocular pressure in the anterior and posterior chambers increased during the NCT test for both Case 3 and Case 4 (see in Fig. 6), with values of the order of 2.6 times higher than the physiological pressure. However, this increment was $12 \%$ lower for the fluid-structure model. The time at peak pressure was $0.73 \mathrm{~ms}$ for the fluid-structure model, whereas for the structural model was only $0.30 \mathrm{~ms}$ (see in table 2), in good agreement with experimental observations.

While the maximal apical displacement was smaller for the FSI model, both simulation strategies (Case 3 and Case 4) showed similar qualitative NCT signals (see in Fig. 7.a-b). In particular, both applanation signals during inward and outward motion occurred at similar time frames and, remarkably, Case 4 showed similar 1st and 2nd applanation lengths (see in Fig. 7), as it occurs with the experimental range (see in Fig. 1). Same observation is valid for the maximum deformation amplitude. Most of corneal biomarkers were similar between the structural and FSI model with the exception of the displacement amplitude (DA) and the corneal hysteresis, with the latter about 3.5 times smaller for the FSI, being close to the experimental measurements (table 2).

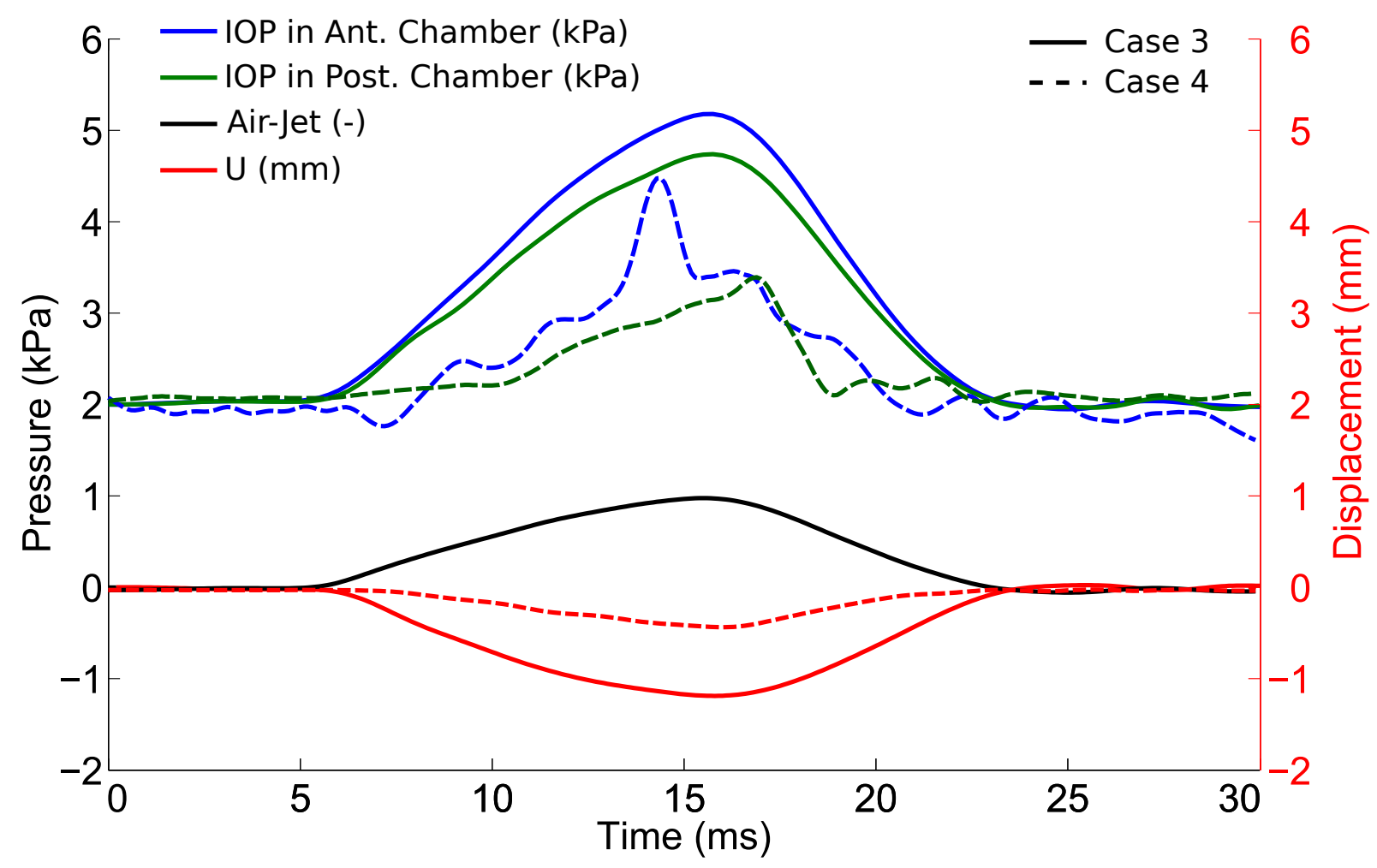

Figure 6: Increment of the Intraocular Pressure (IOP) in the anterior and posterior eye chambers due to the compression of the cornea during the air pulse. The structural model (Case 3, solid lines) resulted in a pressure up to 2.6 times higher than the initial IOP, while the fluid-structure model (Case 4, dashed lines) was up to 2.3 times the intial IOP. 


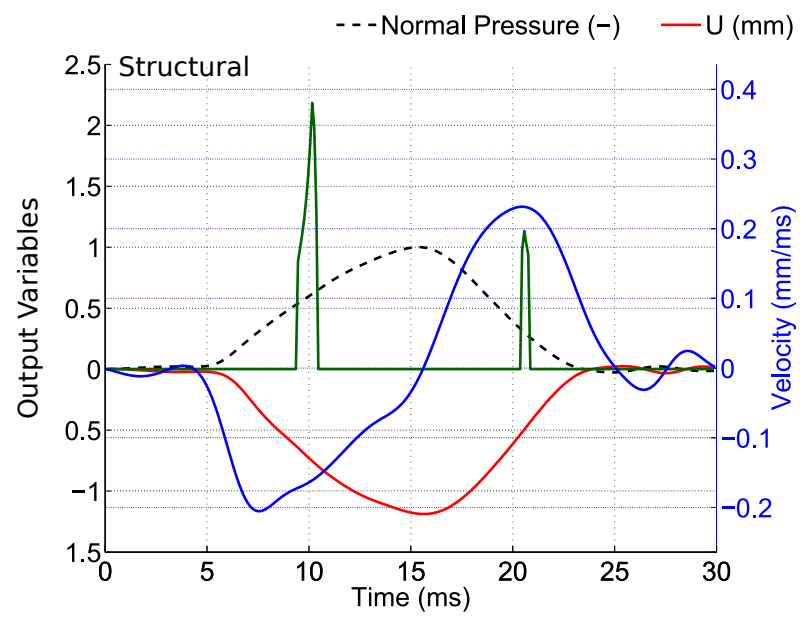

(a.1)

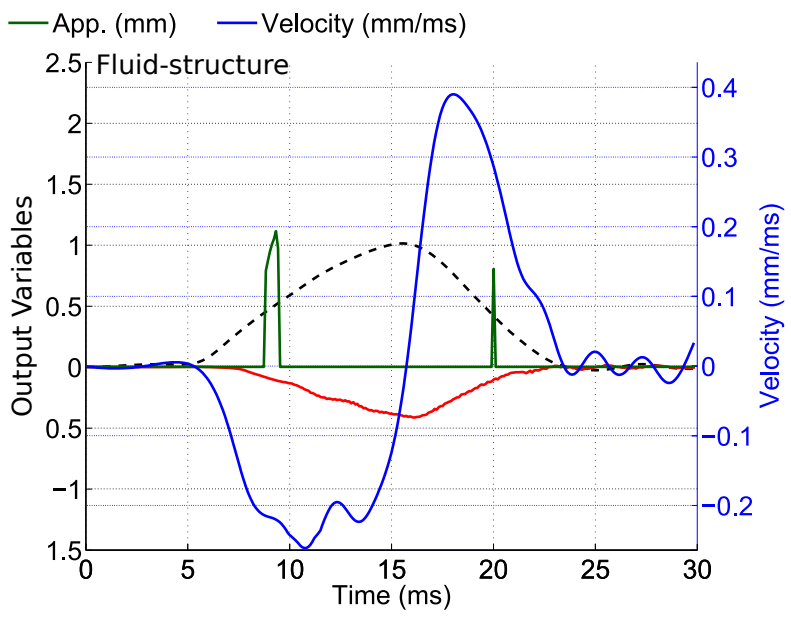

(a.2)

Figure 7: Dynamic explicit simulation of CorVis ST.. (a) Structural simulation (Abaqus Explicit), and (b) Fluid-structure interaction simulation (LS-DYNA). Temporal evolution of corneal biomarkers: apical displacement (red solid line), applanation length (green solid line), velocity (blue solid line), and normalized air pulse pressure (black dashed line, see Fig. 4.b).

Analysis of the internal and kinetic energies along the simulation indicates that, for all structural models (Case 1 through Case 3), the ratio between the kinetic energy and the internal energy was lower than a $1 \%$ indicating that inertial effects do not dominate the overall behavior of the simulation (see Fig. 8.a) and, thus, it could be neglected. When considering Case 4 the overall kinetic energy calculated for the cornea, sclera, and ciliary muscle was slightly higher, but remained very low $(<0.5 \%)$ and could also be neglected (see Fig. 8.b). However, for the lens $(\approx 6 \%)$, the aqueous humor $(\approx 16 \%)$, and the vitreous humor $(\approx 1200 \%)$ inertia was clearly dominating (Fig. 8.c).

Important differences have been found in the air pressure distribution on the cornea over time between the FSI and that from the CFD simulation used for the structural analysis. The maximum pressure developed at the apex was similar for both simulations i.e., about $9 \mathrm{kPa}$. However, during the air-puff, zones with negative pressure developed in the proximities of the limbus in the FSI that were not present in the CFD simulation (Fig. 9.a). The distribution of the average speed over the cornea also supports this finding (Fig. 9.b). During the air-puff, the air-jet remains in contact with the cornea most of the time, with flow detachment occurring at about $5 \mathrm{~mm}$ from the apex leading to the negative pressure shown in Fig.9.a. Also, the pressure distribution in the FSI simulation was found to be significantly narrower than that in the CFD. In addition, the time variation of the peak pressure was much faster in the FSI as compared to the CFD case. Panels a and b in Fig. 10 show that between $12.5 \mathrm{~ms}$ and $15 \mathrm{~ms}$ (time at which the peak pressure occurs) the pressure at the apex increases a $120 \%$ for the FSI and a $72 \%$ for the CFD simulation. The same situation happens during ari-jet deceleration (after 15ms) when, the decay in the apical pressure is more significant for the FSI (a 65\% reduction in $2.5 \mathrm{~ms}$ ) than for the CFD (a $15 \%$ in $2.5 \mathrm{~ms}$ ). 


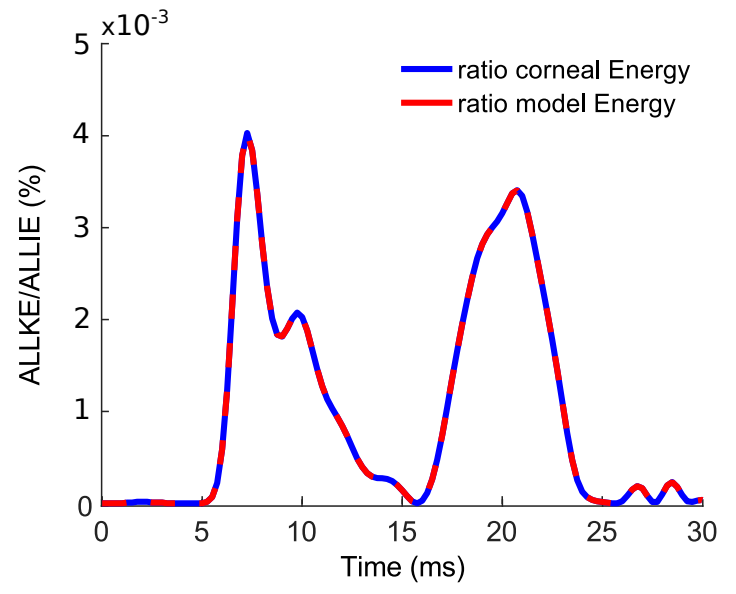

(a)

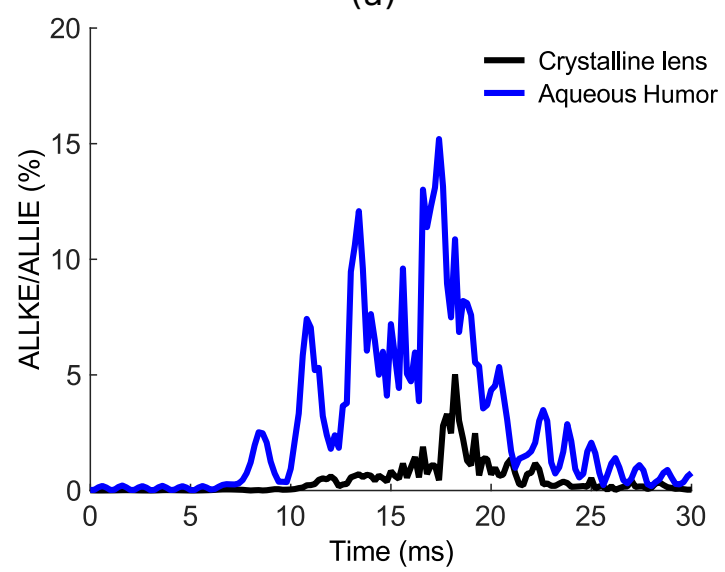

(c)

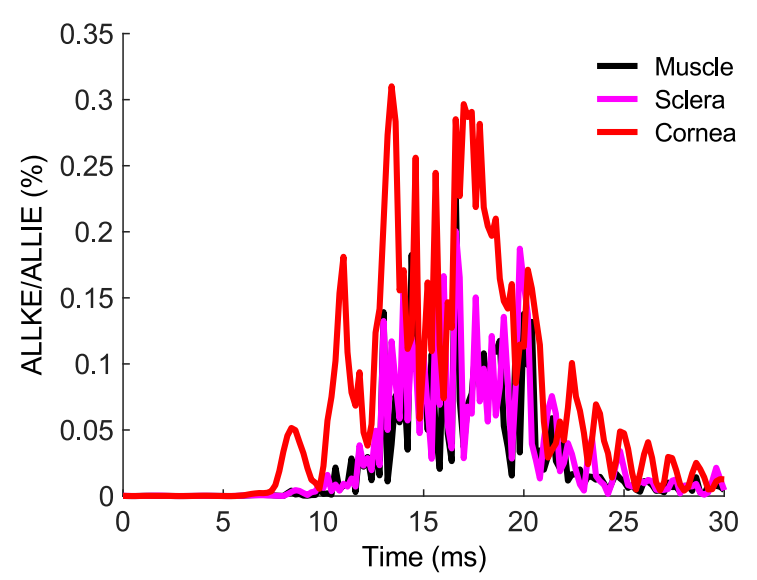

(b)

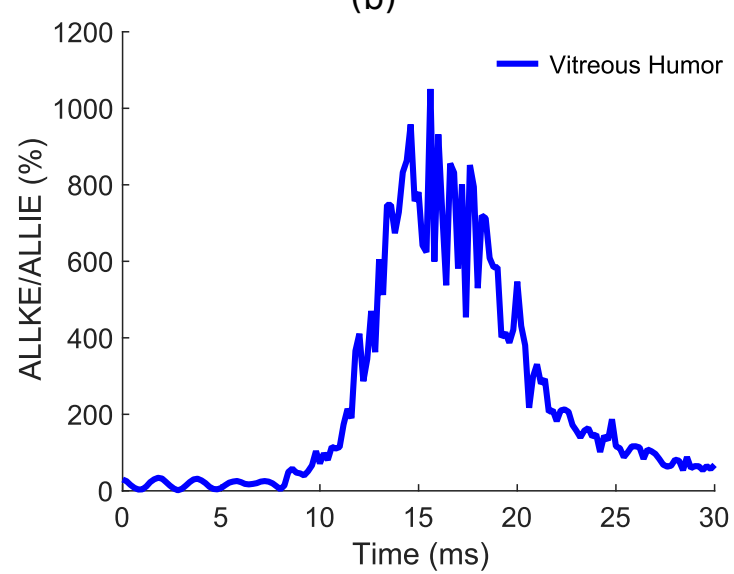

(d)

Figure 8: Ratios of kinetic energy and internall energy in the simulation of a NCT. (a) Structural simulation (case 3). Energies of the cornea (red) and the system (blue); (b) Fluid-structure simulation (case 4). Energies of the structural elements (cornea, sclera, and muscle) are not dominated by the inertia; (c) Fluid-structure simulation (case 4). Energies of the crystalline lens (black) and the aqueous humour (blue) are dominated by the inertia; (d) Fluid-structure simulation (case 4). Energies of the vitreous humour (blue) is dominated by the inertia. 


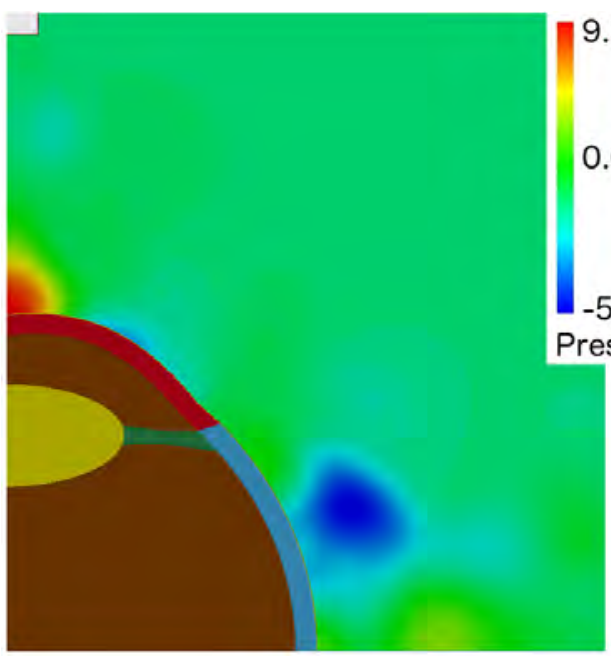

(a)
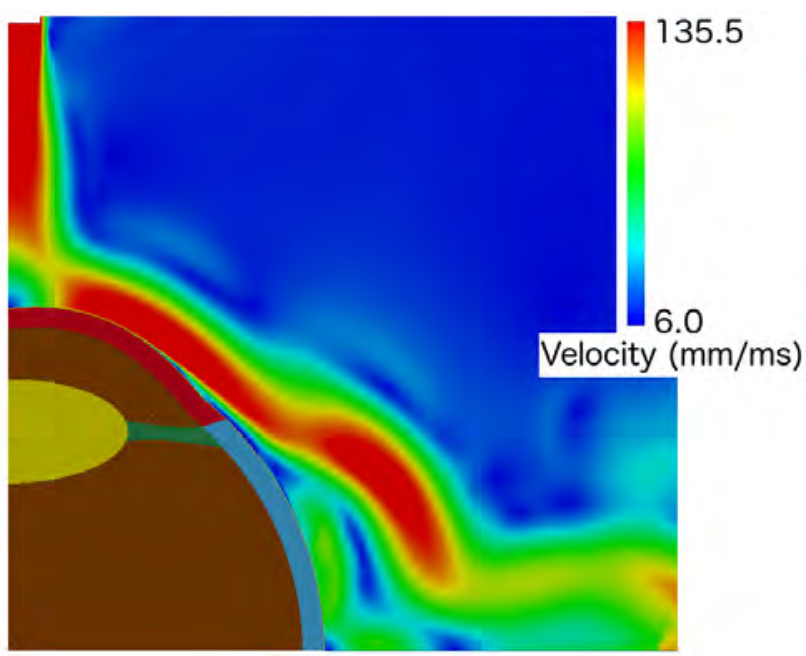

(b)

Figure 9: Analysis of the air domain and the air-cornea interface. Simulation of the Fluid Domain in LS-DYNA $(t=15 \mathrm{~ms}):$ (a) distribution of pressure over the cornea. A peak pressure of $9 \mathrm{kPa}$ is achieved at the corneal center while a negative pressure of up to $2.25 \mathrm{kPa}$ was observed on cornea, at a location between the apex and the sclera (see black arrow); (b) Velocity of the air-jet over the cornea. Recirculation can be observed on the periphery of the cornea, which explains the negative pressure observed at this location. A maximal velocity of $120 \mathrm{~m} / \mathrm{s}$ was reached at the tip of the nozzle.

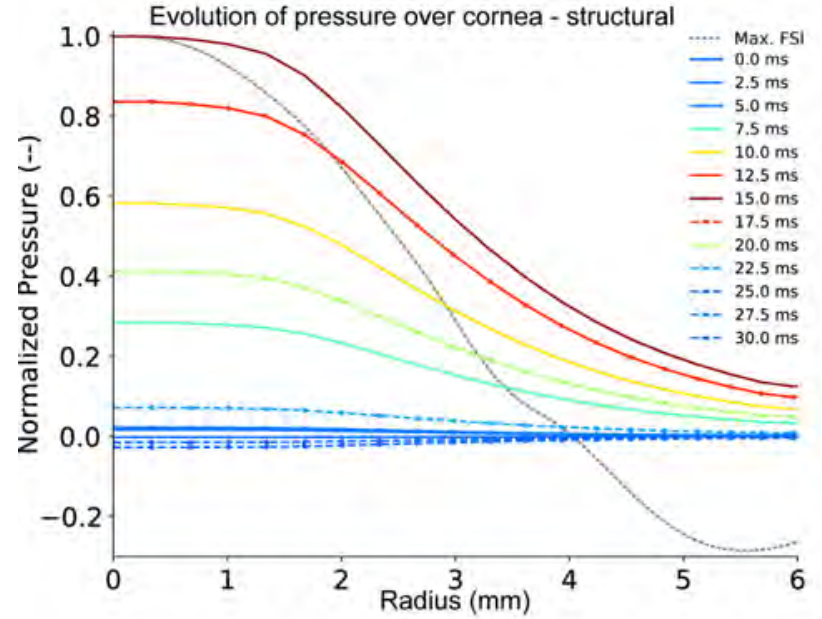

(a)

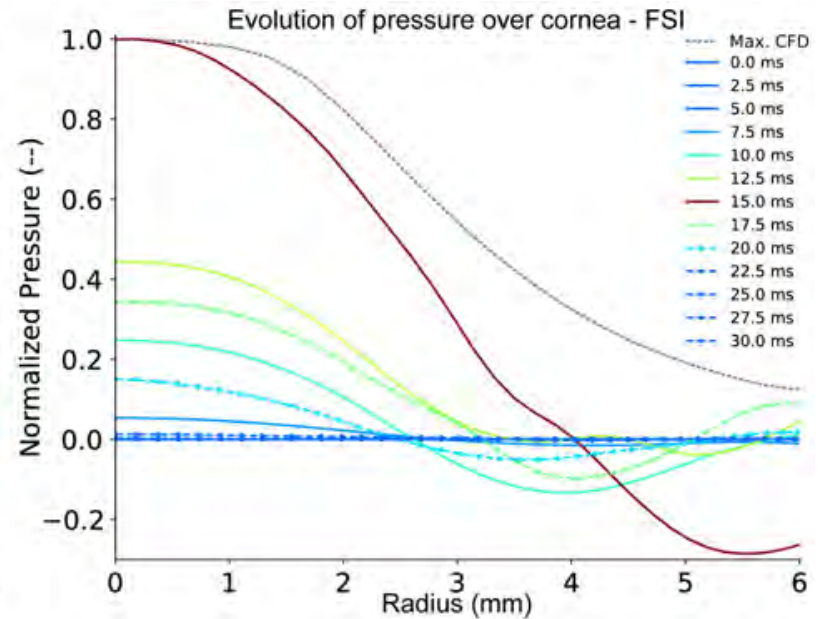

(b)

Figure 10: Pressure distribution at the air-cornea interface for the structural and the FSI simulations: (a) Pressure imposed as boundary condition in the structural model. Peak pressure distribution was calculated with a CFD analysis and, then, escalated using the temporal evolution provided by Oculus (see in Fig. 4.c); (b) Pressure distribution determined by the interaction of the air and the cornea in the FSI simulation. The color scale is the same for both (a and b), but escalates differently depending on the pressure evaluated at the same time frame. 


\section{Discussion}

Non contact tonometry (or air-puff tonometry) is becoming a useful tools not only for the determination of the IOP, but also for studying the mechanical properties of corneal tissue and the evaluation and prognosis of eye pathologies. The physical phenomena behind a NCT test is complex and no consensus exists on which is the most appropriate modeling approach of the problem. In the present work, we have presented a systematic study that analyzes different strategies for modeling the NCT test ranging from pure structural dynamic simulations to full fluid structure interaction simulation. To the best of our knowledge, this is the first FSI simulation including both the air and humors as fluids. Our findings support the need of conducting FSI simulations for a correct modeling of the NCT test.

Our results indicate that assuming a bell-shape distribution for the pressure on the anterior surface of the cornea to simulate the effect of the air-puff is not accurate. The deformation of the cornea during the air-puff facilitates the detachment of the flow from the cornea, causing the development of an area of negative pressure that is not otherwise present if the cornea is simulated as a rigid surface, as commonly happens in CFD simulations. In addition, the deformation of the cornea also causes a narrower spatial pressure profile as compared to the CFD case (see panels $a$ and $b$ in Fig. 10). The combination of these two elements causes that the overall force transmitted to the cornea by the air-puff is lower for the FSI case as compared to the force transmitted by the pressure profile obtained from CFD simulations commonly used in structural simulations $[6,12,13,15]$ with the consequence of a lower apex displacement. As the deformation of the cornea is an interplay of different factors such as geometry, intraocular pressure, and corneal stiffness, it is not possible to know how the spatial distribution of pressure will vary on each patient beforehand, indicating the need of conducting FSI simulations in order to accurately determine the force transmitted to the cornea during the test.

Our findings also demonstrate that considering a uniform pressure in the anterior and posterior cameras of the eye-ball during the simulation of the NCT test is inaccurate. Since the cameras are filled with liquid, the volume must remain constant during the deformation resulting in an increment of the internal pressure when the cornea deforms due to the air-puff. In fact, considering the humors as fluid cavities (without mass) in the structural analysis, or fluid with mass in the FSI, results in an increment of the IOP close to 2-3 times the physiological IOP, in agreement with [6]. This implies that, not accounting for the incompressibility of the humors significantly affect the maximum displacement of the cornea, which results overestimated if a constant pressure is used otherwise. The implications when the mechanical properties of the cornea are to be extrapolated from the test are clear. Neglecting the incompressibility of the humors will result in an overestimation of corneal stiffness. In addition, our findings indicate that internal structures of the eye should be incorporated in the model in order to capture the right behavior of dynamic biomarkers, as they modify the stiffness of the system and slightly change the overall kinematics. Accounting for the lens and ciliary muscles reduces the radial expansion of the eye ball during the air-puff with respect to models that do not include them. In this regard, proper characterization of the stiffness of internal structures and sclera is important as they modulate the overall deformation of the eyeball during the test. In particular, the stiffness of internal structures and sclera significantly impact the increase in intraocular pressure during the test (the more compliant the eyeball, the less increment in intraocular pressure).

Another important consideration when modeling the humors, is to account for their mass. Humors have been traditionally simulated as a distributed uniform pressure without mass [13, 24] or as lumped nodal masses in the back of the corneal surface $[12,15,16]$. However, adding lumped masses is not straightforward, is highly dependent on the volume of the chambers, and could not present a realistic boundary condition unless it is accurately defined. Recently, Montanino et al. [6] outlined the importance of considering the internal humors as a fluid with mass. Results from our FSI simulation in Fig. 8c-d support the findings from Montatino et al. indicating that proper modeling of a NCT test must consider dynamic effects. The maximum deformation amplitude obtained with the FSI simulation was three times smaller (see Case 4 in table 2) as that obtained with a fully structural model of the same characteristics (Case 3). As the structural features between models were the same (material stiffness, internal pressures, and geometry), the observed difference can be attributed to two possible factors. First, a much higher energy will be required to produce the same DA in the FSI model due to the larger inertia in the FSI model associated with the mass of the humors. Secondly, the deformation of the cornea during the air-puff significantly affects the total force transmitted to the cornea with respect to a situation in which the cornea remains fixed during the air-puff, as pointed above. In this particular, our results indicate that not accounting for corneal deformation when simulating the air-puff overestimates the transmitted force to the cornea. 
Unfortunately, we cannot compare our results against other fluid-structure simulations of NCTs as, to the best of our knowledge, there are not other reports available in the literature apart from Montatino et al. [6] that includes the humors but does not account for interaction with the air-puff. . Experimentally, only information about the maximum apical displacement in porcine corneas has been reported [24]. Thus, the rest of corneal biomarkers were compared with respect to those reported in humans [28, 29]. In this regard, we have hypothesized that, at the macroscale, porcine and healthy human eyeballs should behave similarly as a whole system since both present similar anatomical features, masses, and intraocular pressures [32]. In this regard, corneal biomarkers obtained for both simulation (Case 3 and Case 4) strategies were within the order of magnitude of those experimental ranges reported in humans [28, 29]. $\mathrm{CH}$ was fairly out of the experimental range $(22.5 \mathrm{mmHg})$ for the structural simulation, whereas it was three times lower and close to the experimental range $(6.2 \mathrm{mmHg})$ for the fluid-structure simulation. Also, the maximum deformation amplitude (DA) for the fluid-structure simulation was three times smaller than for the structural simulation. Additionally, applanation times were slightly out of the reported ranges and there was a time delay at which the maximum DA was achieved due to the inertia of the system. All these findings indicate that, the inertia of the humors, the increment of pressure in the chambers, and the force transmission at the air-cornea interface are essential features that affect corneal biomarkers associated with a NCT test. In addition, the quantitative similarity between the biomarkers computed in-silico for porcine corneas with experimental results for healthy human corneas, which are anatomically similar [32], suggests that corneal biomarkers are more influenced by the inertia and pressure rather than the morphology of the eye, or the viscoelasticity of the corneal tissue. In fact, Simonini et al. [15] and Montanino et al. [6] suggested that the elasticity of the tissues or the mass of the humors have a greater impact in corneal biomarkers measured with NCTs rather than the viscoelasticity of the tissues itself.

Our study is not exempt of limitations. Anisotropy was not explicitly modeled as our approach is axisymmetric and focuses on evaluating different modeling strategies to simulate a NCT test. Besides, the structural model (case 3) was used to calibrate the material model due to its lower computational cost (i.e., $<10$ minutes for the structural simulation vs. $\approx 4$ hours for the FSI simulation), although fluid-structure simulations (case 4) are expected to be the most realistic. For the sake of simplicity and since our research's objective is not the material behavior, all involved tissues were considered as quasi-incompressible hyperelastic isotropic (cornea, sclera, lens, and muscles)[6, 19]. Moreover, the surrounding boundary conditions have been disregarded, e.g., fat tissue, extraorbital muscles, or optical nerve. The exclusion of the ocular socket could result in inaccurate outcomes as there is a non-negligible displacement of the eyeball inside the ocular cavity [6]. In addition, the viscoelastic behavior of the fat tissue surrounding the eyeball and the extraocular muscles damp the eyeball and allow it to move slighlty backward [12]. Also, the viscoelastic behavior of the cornea has not been taken into account since it has been suggested that the elastic response of the tissue dominates in this high-speed test $[15,16]$. Furthermore, our results suggest that some corneal biomarkers such as the corneal hysteresis appears to have a stronger correlation with the mass and variation of pressure of the internal humors rather than with the viscoelastic response of the cornea itself, an observation already reported in literature [6]. Despite these simplifications, there is no loss of generality in our analysis while the CPU time is greatly reduced. General conclusions regarding the modeling and simulation of NCT remain valid, since we minimized additional bias by using the same material properties and boundary conditions with exemption to those related intrinsically with the simulation, such as the air-jet.

Altogether, our results outline the importance of a proper modelling of the intraocular pressure (IOP) and the forces transmitted to the cornea by the air-puff. In this regard, we have demonstrated that the pressure applied on the anterior surface of the cornea by the air-puff depends on the corneal deformation itself and, therefore the measured mechanical response of the cornea during a NCT test. Our results reinforced our previous results that, corneal deformation results from an interplay between IOP, geometry, and material, but also by internal structures as the humors. Hence, results provided by these devices should be handled with care since they are highly biased by the effect of the internal humors, and not only by the corneal stiffness $[9,14,13]$. Based on our results, we strongly recommend to use fluid-structure simulations, including the internal structures, and modeling the humors as pressurized fluids with mass when modeling an NCT test. Neglecting the use of fluid-structure simulations when performing a material parameter identification would lead to an overestimated corneal stiffness. Last but not least, accurate modeling of NCT test in combination with reliable clinical data could shed light on the mechanisms associated with the mechanical behavior of corneal tissue, but also to help on the development of novel treatments and to improve diagnosis and prognosis of eye pathologies. 


\section{Acknowledgements}

This work was supported by the Spanish Ministry of Economy and Competitiveness (Projects DPI2014-54981-R and DPI2017-84047-R). M. Á. Ariza-Gracia was supported by the Swiss Government through the ESKAS program (ESKAS-No: 2016.0194. Federal Commission for Scholarships for Foreign Students FCS, Switzerland). 


\section{References}

[1] A. Kotecha, A. Elkarmouty, C. Ajtony, K. Barton, Interobserver agreement using Goldmann applanation tonometry and dynamic contour tonometry: comparing ophthalmologists, nurses and technicians., Br. J. Ophthalmol. 100 (6) (2016) 854-9. doi:10.1136/bjophthalmol-2015307219.

[2] D. Weibel, B. Parviz, Contact lens for measuring intraocular pressure. Patent No. US9289123 B2 (mar 2016).

[3] C. D. Giacinto, R. D’Aloisio, S. Trovarelli, A. Accardo, O. Vattovani, D. Tognetto, Rebound Tonometry in Cataract Surgery: Comparison with Goldmann Applanation Tonometry, Open J. Ophthalmol. 08 (01) (2018) 1-11. doi:10.4236/ojoph.2018.81001.

[4] D. P. Piñero, N. Alcón, In vivo characterization of corneal biomechanics, J. Cataract Refractive Surg. 40 (6) (2014) $870-887$. doi:10.1016/j.jcrs.2014.03.021.

[5] D. P. Piñero, N. Alcón, Corneal biomechanics: A review, Clin. Exp. Optom. 98 (2) (2015) 107-116. doi:10.1111/cxo.12230.

[6] A. Montanino, M. Angelillo, A. Pandolfi, Modelling with a meshfree approach the cornea-aqueous humor interaction during the air puff test, J. Mech. Behav. Biomed. Mater. 77 (March 2017) (2018) 205-216. doi:10.1016/j.jmbbm.2017.05.042.

[7] I. B. Pedersen, S. Bak-Nielsen, A. H. Vestergaard, A. Ivarsen, J. Hjortdal, Corneal biomechanical properties after LASIK, ReLEx flex, and ReLEx smile by Scheimpflug-based dynamic tonometry, Graefe's Arch. Clin. Exp. Ophthalmol. 252 (8) (2014) $1329-1335$. doi:10.1007/s00417-014-2667-6.

[8] J. L. Alió, Keratoconus, Springer, 2017. ISBN-9783319438795

[9] M. Á. Ariza-Gracia, J. F. Zurita, D. P. Piñero, J. F. Rodriguez-Matas, B. Calvo, Coupled Biomechanical Response of the Cornea Assessed by Non-Contact Tonometry. A Simulation Study, PLoS One 10 (3) (2015) e0121486. doi:10.1371/journal.pone.0121486.

[10] D. A. Luce, Non-contact tonometry method. United States Patent. Patent Number: US 6,419,631 B1 (2000).

[11] S. Kling, I. B. Akca, E. W. Chang, G. Scarcelli, N. Bekesi, S.-H. Yun, S. Marcos, Numerical model of optical coherence tomographic vibrography imaging to estimate corneal biomechanical properties., J R Soc Interface 11 (101) (2014) 20140920. doi:10.1098/rsif.2014.0920.

[12] A. Sinha Roy, M. Kurian, H. Matalia, R. Shetty, Air-puff associated quantification of non-linear biomechanical properties of the human cornea in vivo, J. Mech. Behav. Biomed. Mater. 48 (APRIL) (2015) 173-182. doi:10.1016/j.jmbbm.2015.04.010.

[13] M. Á. Ariza-Gracia, S. Redondo, D. P. Llorens, B. Calvo, J. F. Rodriguez-Matas, A predictive tool for determining patient-specific mechanical properties of human corneal tissue, Comput. Methods Appl. Mech. Eng. 317 (2016) 226-247. doi:10.1016/j.cma.2016.12.013.

[14] M. Á. Ariza-Gracia, J. Zurita, D. P. Piñero, B. Calvo, J. F. Rodríguez-Matas, Automatized Patient-Specific Methodology for Numerical Determination of Biomechanical Corneal Response, Ann. Biomed. Eng. 44 (5) (2016) 1753-1772. doi:10.1007/s10439-015-1426-0.

[15] I. Simonini, A. Pandolfi, The influence of intraocular pressure and air jet pressure on corneal contactless tonometry tests., J Mech Behav Biomed Mater 58 (2016) 75-89. doi:10.1016/j.jmbbm.2015.07.030.

[16] I. Simonini, M. Angelillo, A. Pandolfi, I. S. M. A. A. Pandolfi, Theoretical and numerical analysis of the corneal air puff test, J. Mech. Phys. Solids 93 (2016) 118-134. doi:10.1016/j.jmps.2016.04.012.

[17] A. Karimi, R. Razaghi, M. Navidbakhsh, T. Sera, S. Kudo, Computing the stresses and deformations of the human eye components due to a high explosive detonation using fluid-structure interaction model, Injury 47 (5) (2016) 1042-1050. doi:10.1016/j.injury.2016.01.030.

[18] A. Karimi, R. Razaghi, M. Navidbakhsh, T. Sera, S. Kudo, Quantifying the injury of the human eye components due to tennis ball impact using a computational fluid-structure interaction model, Sport. Eng. 19 (2) (2016) 105-115. doi:10.1007/s12283-015-0192-4.

[19] S. Salimi, Dynamic Response of Intraocular Pressure and Biomechanical Effects of the Eye Considering Fluid-Structure Interaction, J. Biomech. Eng. 133 (9) (2011) 091009. doi:10.1115/1.4005166.

[20] O. H. Yeoh, Some Forms of the Strain Energy Function for Rubber, Rubber Chem. Technol. 66 (5) (1993) 754-771. doi:10.5254/1.3538343.

[21] M. Á. Ariza-Gracia, Á. Ortillés, J. Cristóbal, J. F. Rodríguez Matas, B. Calvo, A Numerical-Experimental Protocol to Characterize Corneal Tissue with an Application to Predict Astigmatic Keratotomy Surgery, J. Mech. Behav. Biomed. Mater. 74 (February) (2017) $304-314$. doi:10.1016/j.jmbbm.2017.06.017.

[22] H. Demiray, A note on the elasticity of soft biological tissues, J. Biomech. 5 (3) (1972) 309-311.

[23] F. Boschetti, A. Pandolfi, Biomechanical Properties of Porcine Corneas, J. Biomchanics 45 (2012) 2012. doi:10.1016/S0021-9290(12)705791 .

[24] S. Kling, N. Bekesi, C. Dorronsoro, D. Pascual, S. Marcos, Corneal viscoelastic properties from finite-element analysis of in vivo air-puff deformation., PLoS One 9 (8) (2014) e104904. doi:10.1371/journal.pone.0104904.

[25] D. C. Montgomery, Design and Analysis of Experiments, John Wiley \& Sons, Inc., Hoboken, NJ, 2002. doi:10.1002/qre.458.

[26] G. Van Rossum, F. L. Drake, The Python language reference manual : for Python version 3.2, Network Theory Ltd, 2011.

[27] A. S. Seetamsetti, Comparison of Finite Element Analysis of Impact on Water and Soil Using Lagrangian, ALE and SPH Approaches and Airframe Impact Applications, Ph.D. thesis, Graduate School of Wichita State University (2012).

[28] Y. Hon, A. K. C. Lam, Corneal deformation measurement using Scheimpflug noncontact tonometry., Optom Vis Sci 90 (1) (2013) e1-e8. doi:10.1097/OPX.0b013e318279eb87.

[29] D. A. Luce, Determining in vivo biomechanical properties of the cornea with an ocular response analyzer, J. Cataract Refract. Surg. 31 (1) (2005) 156-162. doi:10.1016/j.jcrs.2004.10.044.

[30] G. Labiris, M. Gkika, A. Katsanos, M. Fanariotis, E. Alvanos, V. Kozobolis, Anterior chamber volume measurements with Visante optical coherence tomography and Pentacam: repeatability and level of agreement, Clin. Experiment. Ophthalmol. 37 (8) (2009) $772-774$. doi:10.1111/j.1442-9071.2009.02132.x.

[31] J. V. Forrester, A. D. A. D. Dick, P. G. McMenamin, F. P. Roberts, E. Pearlman, The eye : basic sciences in practice, 4th Edition, Elsevier, 2015.

[32] I. Sanchez, R. Martin, F. Ussa, I. Fernandez-Bueno, The parameters of the porcine eyeball, Graefe's Arch. Clin. Exp. Ophthalmol. 249 (4) (2011) 475-482. doi:10.1007/s00417-011-1617-9.

[33] J. C. Simo, On a fully three-dimensional finite-strain viscoelastic damage model: formulation and computational aspects, Comput. Methods Appl. Mech. Eng. 60 (2) (1987) 153-173. 
[34] S. J. Petsche, P. M. Pinsky, The role of 3-D collagen organization in stromal elasticity: A model based on X-ray diffraction data and second harmonic-generated images, Biomech. Model. Mechanobiol. 12 (6) (2013) 1101-1113. doi:10.1007/s10237-012-0466-8. 